\title{
Modelling of Graphene Nanoribbon Fermi Energy
}

\author{
Zaharah Johari, Mohammad Taghi Ahmadi, Desmond Chang Yih Chek, \\ N. Aziziah Amin, and Razali Ismail
}

Department of Electronic Engineering, Faculty of Electrical Engineering, Technological University of Malaysia, Skudai, 81310 Johor Darul Takzim, Malaysia

Correspondence should be addressed to Mohammad Taghi Ahmadi, ahmadiph@gmail.com

Received 30 December 2009; Accepted 16 March 2010

Academic Editor: Rakesh Joshi

Copyright ( 2010 Zaharah Johari et al. This is an open access article distributed under the Creative Commons Attribution License, which permits unrestricted use, distribution, and reproduction in any medium, provided the original work is properly cited.

Graphene nanoribbon (GNR) is a promising alternative to carbon nanotube (CNT) to overcome the chirality challenge as a nanoscale device channel. Due to the one-dimensional behavior of plane GNR, the carrier statistic study is attractive. Research works have been done on carrier statistic study of GNR especially in the parabolic part of the band structure using Boltzmann approximation (nondegenerate regime). Based on the quantum confinement effect, we have improved the fundamental study in degenerate regime for both the parabolic and nonparabolic parts of GNR band energy. Our results demonstrate that the band energy of GNR near to the minimum band energy is parabolic. In this part of the band structure, the Fermi-Dirac integrals are sufficient for the carrier concentration study. The Fermi energy showed the temperature-dependent behavior similar to any other one-dimensional device in nondegenerate regime. However in the degenerate regime, the normalized Fermi energy with respect to the band edge is a function of carrier concentration. The numerical solution of Fermi-Dirac integrals for nonparabolic region, which is away from the minimum energy band structure of GNR, is also presented.

\section{Introduction}

Single layer of graphite which is also known as graphene has been discovered as a material with attractive lowdimensional physics, and possible applications in electronics [1-6]. A single-wall carbon nanotube (SWCNT) is a piece of rolled-up graphene sheet while a nanoribbon is an unrolled nanotube. Band-gap opening is expected by patterning narrow ribbons [7, 8] from Graphene which can be achieved by chemical means [9]. This Graphene nanoribbon (GNR) with quasi-one-dimensional structures and narrow widths $(<\sim 10 \mathrm{~nm})$ is predicted to be used as a channel for field effect transistors with high switching speed and excellent carrier mobility with ballistic transport behavior [9-14]. Armchair and zigzag GNRs show metallic or semiconducting electronic properties depending on the number of dimer lines, $N$ which gives the width of the nanoribbon as depicted in Figures 1 and 2. The semiconducting property in armchair GNRs occurs when $N=3 p$ or $3 p+1$, where $p$ is an integer [15]. The width of the GNR, $w_{\mathrm{GNR}}$, is proportional to $N$ given by the expression

$$
w_{\mathrm{GNR}}=(N-1) \frac{\sqrt{3}}{2} a_{\mathrm{C}-\mathrm{C}}
$$

where $a_{\mathrm{C}-\mathrm{C}}=0.142 \mathrm{~nm}$ is the lattice constant [16]. Quantum confinement effect results in similarity of semiconducting and metallic behaviors in both nanotube and nanoribbon configurations. A nanoribbon can be assumed as an unrolled single-wall nanotube that results in two different classes of GNRs depending on SWNTs unfolded way. One is by unzipping the SWNT along the axial direction through a row of atoms and then splitting the atom row onto both edges of the resulting GNR [17].

\section{Graphene Nanoribbon Band Structure}

The band energy throughout the entire Brillouin zone of graphene is given by [18] 


$$
\begin{aligned}
& E(\vec{k})= \\
& \pm t \sqrt{1+4 \cos \left(\frac{k_{x} 3 a_{\mathrm{C}-\mathrm{C}}}{2}\right) \cos \left(\frac{k_{y} \sqrt{3} a_{\mathrm{C}-\mathrm{C}}}{2}\right)+4 \cos ^{2}\left(\frac{k_{y} \sqrt{3} a_{\mathrm{C}-\mathrm{C}}}{2}\right)},
\end{aligned}
$$

where $a_{\mathrm{C}-\mathrm{C}}=1.42 \AA$ is carbon-carbon $(\mathrm{C}-\mathrm{C})$ bond length, $t=2.7 \mathrm{eV}$ is the nearest neighbor $\mathrm{C}-\mathrm{C}$ tight-binding overlap energy, and $k_{x, y, z}$ is the wave vector component $[19,20]$. In low-energy limit [21] due to the approximation for the graphene band structure near the Fermi point, the $E(k)$ relation of the GNR is obtained as

$$
E(\vec{k})= \pm \frac{t 3 a_{\mathrm{C}-\mathrm{C}}}{2} \sqrt{k_{x}^{2}+\beta^{2}}
$$

where $\beta$ is the quantized wave vector [22] given as

$$
\beta=\frac{2 \pi}{a_{\mathrm{c}-\mathrm{c}} \sqrt{3}}\left(\frac{p_{i}}{N+1}-\frac{2}{3}\right),
$$

where $p_{i}$ is the subband index and $N$ is the number of dimmer lines which determine the width of the ribbon [21], and $k_{x}$ is the wave vector along the length of the nanoribbon. The energy band gap of the GNR is then simplified to be

$$
E_{g}=3 t a_{\mathrm{c}-c} \beta
$$

By using (5) as the band gap, we can rewrite the band energy as [22]

$$
E= \pm \frac{E_{g}}{2} \sqrt{\left(1+\frac{k_{x}^{2}}{\beta^{2}}\right)}
$$

According to (6), the relationship between energy and wave vector is not parabolic. In the case of semiconducting GNR, the square root approximation can be employed to formulate the parabolic relation between energy and wave vector. Therefore the band energy in the low-energy limit $(k=0)$ is

$$
\begin{gathered}
E(k) \approx \frac{E_{g}}{2}+\frac{E_{g}}{4 \beta^{2}} k_{x}^{2}, \\
E \approx \frac{E_{g}}{2}+\frac{\hbar^{2} k_{x}^{2}}{2 m^{*}},
\end{gathered}
$$

where $m^{*}$ is the effective mass of GNR. The square root approximation leads to Fermi-Dirac integrals in the parabolic band structure for the carrier concentration, velocity, and current expression which is similar to the onedimensional devices [22]. By representing the GNR energy band of (6) and (8) in Figure 3, it can be seen that the band structure is parabolic at certain range of energy in the $E-k$ relationship. In the parabolic region it is sufficient to use the Fermi-Dirac integral for carrier statistic study. However in the nonparabolic part, it will lead to a different kind of Fermi integral.

By taking the derivatives of energy $E$ over the wave vector $k(d E / d k)$ for (8), the density of state (DOS) [22] for parabolic part of one-dimensional GNR band energy

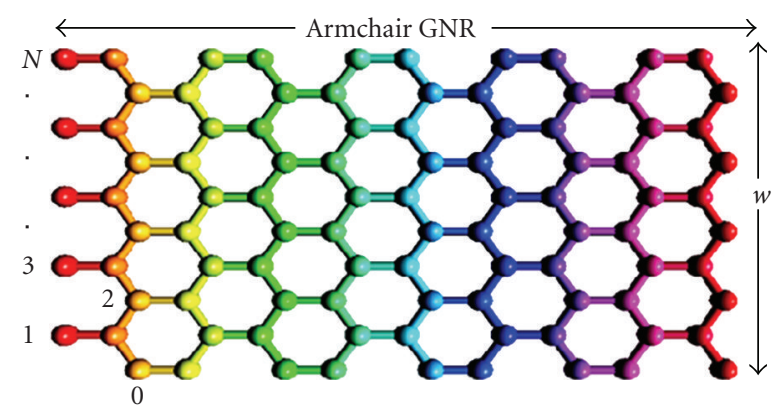

FIGURE 1: Armchair GNRs with dimer lines $N$ and width $w$.

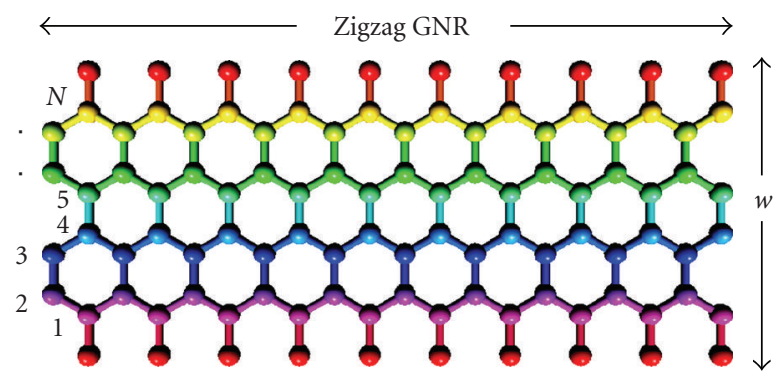

FIgURE 2: Zigzag GNRs with dimer lines $N$ and width $w$.

incorporating the effect of electron-spin parameter $\left(g_{s}=2\right)$ [23] is given by

$$
\operatorname{DOS}_{\mathrm{GNR}}(E)=\frac{\Delta n}{\Delta E \cdot L}=\frac{1}{2 \pi}\left(\frac{2 m^{*}}{\hbar^{2}}\right)^{1 / 2}\left(E-\frac{E_{g}}{2}\right)^{-1 / 2},
$$

where $L$ is the length of the ribbon. By substituting $E_{g} / 2$ with shifted conduction band energy $E_{C}$, this DOS equation can be readily applied to another one-dimensional material.

\section{Result and Discussion}

3.1. Carrier Statistics in Parabolic Band Structure. Carrier concentration is an essential parameter for semiconductor. The numbers of electrons $/ \mathrm{cm}^{3}$ and holes $/ \mathrm{cm}^{3}$ with energies between $E$ and $E+d E$ have been established to be $\operatorname{DOS}(E) f(E) d(E)$ and $\operatorname{DOS}(E)[1-f(E)] d E$.

Therefore the total carrier concentration in a band can be obtained by simply integrating the Fermi-Dirac distribution function over energy band as follows [23]:

$$
\begin{gathered}
n=\int_{E_{C}}^{E_{\text {top }}} \operatorname{DOS}(E) f(E) d E, \\
p=\int_{E_{\text {bottom }}}^{E_{V}} \operatorname{DOS}(E)[1-f(E)] d E .
\end{gathered}
$$

By substituting the density of state $\operatorname{DOS}_{\mathrm{GNR}}(E)$ and FermiDirac distribution function $f(E)$ expressions into carrier concentration, we obtained [24]

$$
n_{D}=N_{C} \mathfrak{I}_{(D-2) / 2}\left(\eta_{D}\right),
$$

where $D$ is dimensionality $D=3(3 \mathrm{D}), 2(2 \mathrm{D})$, and $1(1 \mathrm{D})$, $N_{C}$ is effective density of states, $N_{C}=\left(2 m^{*} k_{B} T / \pi \hbar^{2}\right)^{1 / 2}$, 


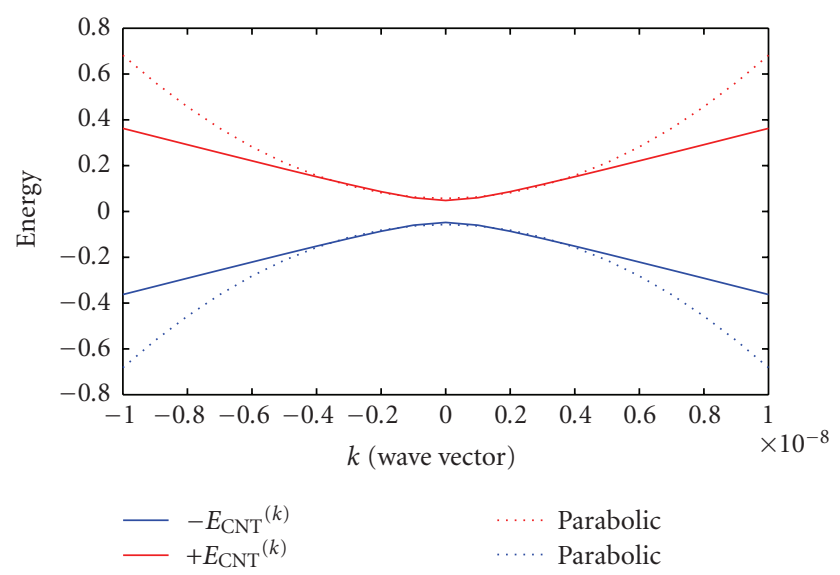

FIGURE 3: The band structure of GNR near the minimum energy is parabolic.

$\mathfrak{I}_{(D-2) / 2}$ is the Fermi-Dirac integral of order $(D-2) / 2$, and normalized Fermi energy is $\eta_{D}=\left(E_{F}-E_{C D}\right) / k_{B} T$. The Fermi-Dirac integral of order $i$ is defined as

$$
\mathfrak{I}_{i}\left(\eta_{D}\right)=\frac{1}{\Gamma(i+1)} \int_{0}^{\infty} \frac{x^{i}}{e^{\left(x-\eta_{D}\right)}+1} d x .
$$

Under nondegenerate condition, the " 1 " from the denominator can be neglected and hence the Fermi integral reduces to

$$
\mathfrak{I}_{i}(\eta) \approx e^{\eta}
$$

In degenerate regime, the exponential part of (14) is very small because all the levels are occupied by electrons up to Fermi level. Thus, the occupation probability is 1 and the Fermi- Dirac integral can be solved analytically resulting in

$$
\mathfrak{I}_{i}\left(\eta_{D}\right)=\frac{1}{\Gamma(i+1)} \frac{\eta_{D}{ }^{i+1}}{i+1} .
$$

The inequalities adjacent to (14) are simultaneously satisfied if the Fermi level lies in the band gap more than $3 K_{B} T$ from either band edge. Figure 4 shows the energy band diagram indicating the degenerate and nondegenerate regions. Conversely, if the Fermi level is within $3 K_{B} T$ of either band edge or lies inside a band, the semiconductor is said to be degenerate. It should be noted that a nondegenerate positioning of the Fermi level makes $f(E) \approx e^{-\left(\left(E-E_{F}\right) / K_{B} T\right)}$ for all conduction band energies and $1-f(E) \approx e^{\left(\left(E-E_{F}\right) / K_{B} T\right)}$ for all valence band energies for all dimensions.

The simplified form of the occupancy factors is a Maxwell-Boltzmann-type function that also describes, for example, the energy distribution of molecules in a high temperature. The simplified occupancy factors lead directly to the nondegenerate relationships. In closed-form relationships we find limited usage in device analysis since the nondegenerate relationships are only valid for an intrinsic and low-doped semiconductor [22]. Quasi-one-dimensional GNR in Figure 5 has two axis ( $x$ and $z$ ) directions that are less than De Broglie wave-length (one layer atomic thickness

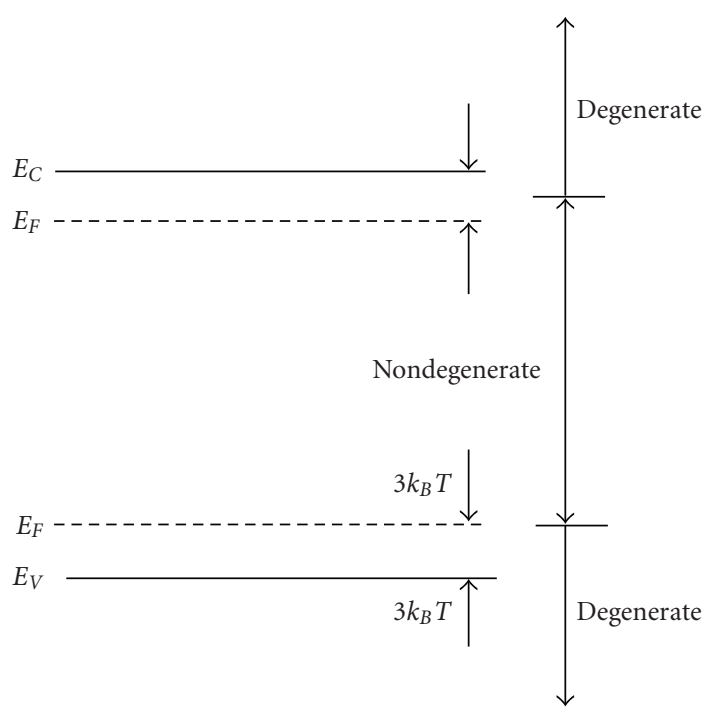

Figure 4: Energy band diagram showing degenerate and nondegenerate regions.

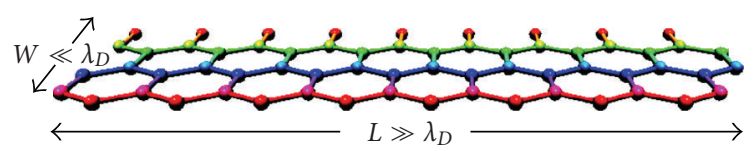

FIGURE 5: Cross-section of a rectangular one-dimensional GNR with $W \ll \lambda_{D}$ and $\gg \lambda_{D}$.

with width less than $10 \mathrm{~nm}$ ). However, GNR has length that is more than De Broglie wave-length, $\mathrm{L} \gg \lambda_{\mathrm{D}}$. The width is less than De Broglie wave-length $\ll \lambda_{\mathrm{D}}$.

In nondegenerate limit condition, the carrier concentration of GNR can be expressed as

$$
n_{\mathrm{GNR}(\text { non deg enerate })}=N_{C} e^{-\left(\left(E_{C}-E_{F}\right) / k_{B} T\right)} .
$$

Here $E_{C}=E_{C 0}+\varepsilon_{0 y}+\varepsilon_{0 z}$ is the shifted conduction band due to the confinement effect. This simplified distribution function is extensively used in determining the transport parameters. It is applicable for nondegenerately doped semiconductors and also GNR near the minimum band energy. However, most nanoelectronic devices today are degenerately doped $[25,26]$. However, any design based on the Maxwellian distribution is not strictly correct and often leads to errors in the interpretation of the results.

For degenerate GNR we have

$$
n_{\mathrm{GNR}(\mathrm{deg})}=\left[\frac{8 m^{*}\left(E_{F}-E_{C}\right)}{\pi^{2} \hbar^{2}}\right]^{1 / 2} .
$$

In quasi-one-dimensional semiconducting GNR, the FermiDirac integral is a proportion of exponential of $\eta$ in nondegenerate approximation and a proportion of $2 / \sqrt{\pi} e^{1 / 2}$ in degenerate approximation as shown in Figure 6. This significant result proves that the Fermi-Dirac integral can be simplified into degenerate and nondegenerate approximation and therefore a simplified equation can be obtained. 


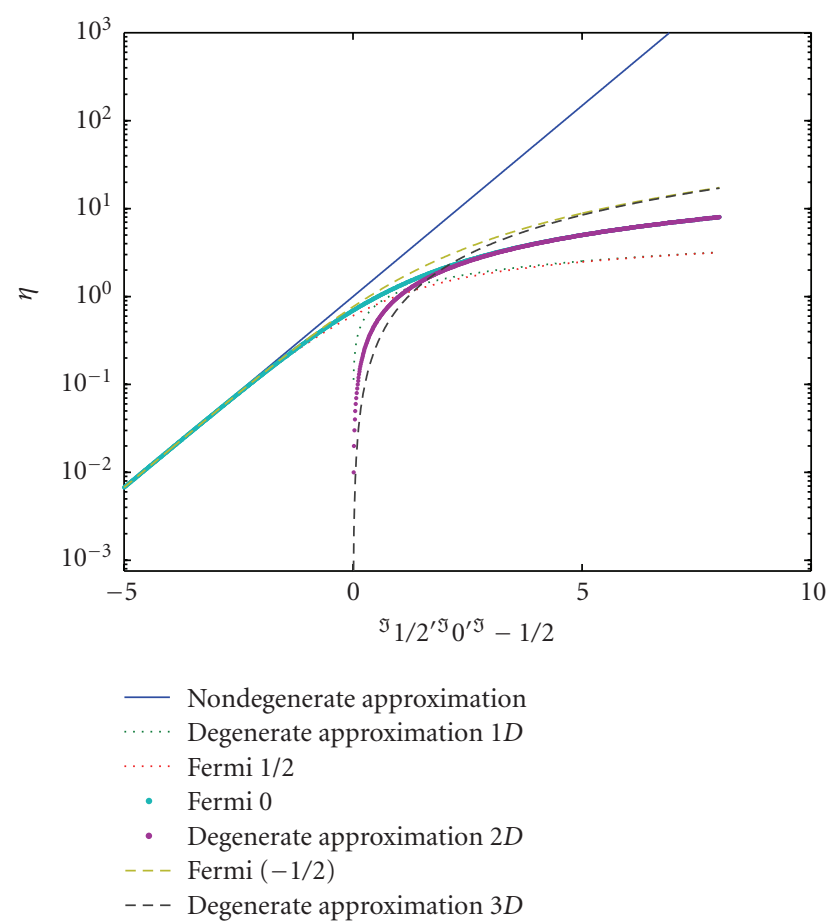

Figure 6: Comparison of Fermi-Dirac integral and Fermi - 1/2, 0, 1/2 for Q3D bulk, Q2D, and Q1D devices, respectively [27].

Also shown in Figure 6 are those for $D=3$ (bulk), 2(quasi-2-dimensional), and 1(quasi-1-dimensional). The normalized Fermi energy $\eta_{F d}$ as a function of normalized carrier concentration in the nondegenerate regime of GNR is given by [24]

$$
\left(E_{F}-E_{C}\right)_{\text {non deg enerate }}=k_{B} T \ln \left(\frac{n}{N}\right)_{d},
$$

where $\left(E_{F}-E_{C}\right)_{\text {non deg enerate }}$ is a weak (logarithmic) function of carrier concentration. It varies linearly with temperature in the nondegenerate (ND) regime. However, for strongly degenerate statistics, the Fermi energy is independent of temperature and it is a strong function of carrier concentration given by [24]

$$
\left(E_{F}-E_{C}\right)_{\text {deg enerate }}=\frac{\hbar^{2}}{m^{*}} 2 \pi\left[\Gamma\left(\frac{d}{2}+1\right) n_{d} / 2\right]^{2 / d} .
$$

The Fermi energy is proportional to $n_{1}^{2}$ for 1D nanostructure specifically for the GNR. Equations (18) and (19) are important as we would like to know the position of the Fermi level with respect to the carrier concentration in GNR. The higher doping concentration on GNR will lead to higher carrier concentration and hence Fermi level is further from conduction band.

3.2. Carrier Statistics in Nonparabolic Band Structure. As illustrated in Figure 3, the energy band is only parabolic near the low-energy limit in a range of $-0.5<k<0.5$. The non-parabolic part remains unsolved outside that range.

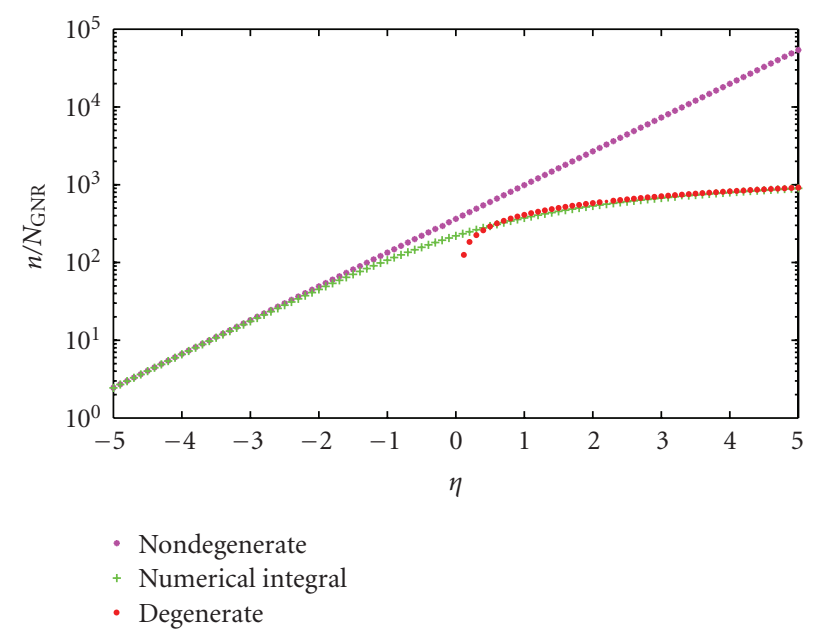

FIgure 7: Comparison of the GNR Fermi-Dirac integral in degenerate and nondegenerate regimes in non-parabolic region. Also shown is the comparison with general equation ( $W=3.5663$, $m=10, p=21$, and $N=30$ ).

The carrier concentration in the non-parabolic region is determined by

$$
n_{\mathrm{GNR}}=\int_{-\infty}^{\infty} \frac{1}{3 \pi a_{c-c} t} \frac{E}{\sqrt{E^{2}-\left(E_{g} / 2\right)^{2}}} \frac{1}{e^{\left(E-E_{F}\right) / k_{B} T}+1} d E .
$$

However, this integral cannot be solved analytically. Rearranging $(20)$ we obtained

$$
n_{\mathrm{GNR}}=N_{C} \int_{-\infty}^{\infty} \frac{\left(x+E_{g} / 2 k_{B} T\right)}{\sqrt{x^{2}+x\left(E_{g} / k_{B} T\right)}}\left(\frac{1}{e^{x-\eta_{\mathrm{FGNR}}}+1}\right) d x,
$$

where $N_{C}=k_{B} T / 3 \pi a_{c-c} t, x=E-\left(E_{g} / 2\right) / k_{B} T$, and $\eta_{F G N R}=$ $E_{F}-\left(E_{g} / 2\right) / k_{B} T . N_{C}$ is the effective density of states in the nonparabolic region of the GNR. For the nondegenerate condition which neglected 1 in the denominator, (21) will be similar to the other one-dimensional devices which showed temperature dependence effect for the Fermi energy. By replacing the graphene nanoribbon Fermi integral with another integral $M$, the equation for electron concentration in non-parabolic region of the GNR will be simplified as

$$
n_{\mathrm{GNR}}=N_{C} M e^{\eta_{\mathrm{GNR}}},
$$

where

$$
M=\int_{E_{C}}^{E_{\mathrm{top}}} \frac{x+E_{G} / 2 k_{B} T}{\sqrt{x^{2}+x E_{G} / k_{B} T}}\left(\frac{1}{e^{x-\eta}+1}\right) d x .
$$

As the carrier occupancy probability is unity in the degenerate regime, the exponential part can be neglected, which leads to

$$
n_{\mathrm{GNR}}=N_{C} \int_{0}^{\eta} \frac{x+E_{G} / 2 k_{B} T}{\sqrt{x^{2}+x E_{G}} / k_{B} T} d x .
$$


Thus, the carrier concentration is a simple integral which can be solved analytically. Expanding (24)

$$
n_{\mathrm{GNR}}=N_{C} \int_{0}^{\eta} \frac{x}{\sqrt{x^{2}+x E_{G} / k_{B} T}} d x+\int_{0}^{\eta} \frac{E_{G} / 2 k_{B} T}{\sqrt{x^{2}+x E_{G} / k_{B} T}} d x .
$$

By substituting $x$ and $\eta_{F G N R}$ into (25), the normalized Fermi energy is dependent on the carrier concentration and independent of temperature. Thus, the non-parabolic carrier concentration of the GNR can be expressed as

$$
n_{\mathrm{GNR}}=N_{C}\left(\sqrt{\left(E_{F}-E_{C}\right)^{2}+\left(E_{F}-E_{C}\right)^{2} E_{G}}\right)
$$

In quasi-one-dimensional semiconducting nanoribbon the Fermi-Dirac integral is proportional to the exponential of $\eta$ in nondegenerate approximation and it is proportional to carrier concentration in degenerate approximation. Figure 7 shows that the nanoribbon Fermi integral in non-parabolic region is closely approximated by the exponential of $\eta$ when $\eta \leq-3$ for the nondegenerate regime. Thus GNR Fermi energy is a function of temperature that is independent of the carrier concentration in the nondegenerate regime. In the other strongly degenerate regime for $(\eta \geq 6)$, the Fermi energy is a function of carrier concentration appropriate for given dimensionality and it is independent of temperature.

\section{Conclusion}

The modeling of carrier statistic in a both parabolic and nonparabolic region is presented. The one-dimensional GNR approaches degeneracy at relatively lower values of carrier concentration as compared to $2 \mathrm{D}$ and $3 \mathrm{D}$ structures. The Fermi energy with respect to the band edge of the GNR is a function of temperature but is independent of the carrier concentration in the nondegenerate regime. In the strongly degenerate regime, the Fermi energy is a function of carrier concentration but it is independent of temperature.

\section{Acknowledgments}

The authors would like to acknowledge the financial support from FRGS grant of the Ministry of Higher Education (MOHE), Malaysia. Mohammad Taghi Ahmadi is thankful to the Research Management Centre (RMC) of Universiti Teknologi Malaysia (UTM) for providing excellent research environment in which to complete this work.

\section{References}

[1] A. K. Geim and K. S. Novoselov, "The rise of graphene," Nature Materials, vol. 6, no. 3, pp. 183-191, 2007.

[2] K. S. Novoselov, A. K. Geim, S. V. Morozov, et al., "Electric field in atomically thin carbon films," Science, vol. 306, no. 5696, pp. 666-669, 2004.
[3] C. Berger, Z. Song, X. Li, et al., "Electronic confinement and coherence in patterned epitaxial graphene," Science, vol. 312, no. 5777, pp. 1191-1196, 2006.

[4] Y. Zhang, Y.-W. Tan, H. L. Stormer, and P. Kim, "Experimental observation of the quantum Hall effect and Berry's phase in graphene," Nature, vol. 438, no. 7065, pp. 201-204, 2005.

[5] K. S. Novoselov, A. K. Geim, S. V. Morozov, et al., "Twodimensional gas of massless Dirac fermions in graphene," Nature, vol. 438, no. 7065, pp. 197-200, 2005.

[6] C. Berger, Z. Song, T. Li, et al., "Ultrathin epitaxial graphite: $2 \mathrm{D}$ electron gas properties and a route toward graphene-based nanoelectronics," Journal of Physical Chemistry B, vol. 108, no. 52, pp. 19912-19916, 2004.

[7] Y.-W. Son, M. L. Cohen, and S. G. Louie, "Energy gaps in graphene nanoribbons," Physical Review Letters, vol. 97, no. 21, Article ID 216803, 4 pages, 2006.

[8] H. Xiang, E. Kan, S.-H. Wei, M.-H. Whangbo, and J. Yang, "“Narrow" Graphene Nanoribbons Made Easier by Partial Hydrogenation," Nano Letters, vol. 9, no. 12, pp. 4025-4030, 2009.

[9] M. Y. Han, B. Özyilmaz, Y. Zhang, and P. Kim, "Energy bandgap engineering of graphene nanoribbons," Physical Review Letters, vol. 98, no. 20, Article ID 206805, 4 pages, 2007.

[10] D. A. Areshkin, D. Gunlycke, and C. T. White, "Ballistic transport in graphene nanostrips in the presence of disorder: importance of edge effects," Nano Letters, vol. 7, no. 1, pp. 204210, 2007.

[11] Z. Chen, Y.-M. Lin, M. J. Rooks, and P. Avouris, "Graphene nano-ribbon electronics," Physica E, vol. 40, no. 2, Article ID 0701599, pp. 228-232, 2007.

[12] K. Nakada, M. Fujita, G. Dresselhaus, and M. S. Dresselhaus, "Edge state in graphene ribbons: nanometer size effect and edge shape dependence," Physical Review B, vol. 54, no. 24, pp. 17954-17961, 1996.

[13] V. Barone, O. Hod, and G. E. Scuseria, "Electronic structure and stability of semiconducting graphene nanoribbons," Nano Letters, vol. 6, no. 12, pp. 2748-2754, 2006.

[14] G. Liang, N. Neophytou, D. E. Nikonov, and M. S. Lundstrom, "Performance projections for ballistic graphene nanoribbon field-effect transistors," IEEE Transactions on Electron Devices, vol. 54, no. 4, pp. 677-682, 2007.

[15] K. Nakada, M. Fujita, G. Dresselhaus, and M. S. Dresselhaus, "Edge state in graphene ribbons: nanometer size effect and edge shape dependence," Physical Review B, vol. 54, no. 24, pp. 17954-17961, 1996.

[16] K. Wakabayashi, "Electronic and Magnetic Properties of Nanographite," in Carbon-Based Magnetism-n Overview of the Magnetism of Metal Free Carbon-Based Compounds and Materials, pp. 279-304, Elsevier, Amsterdam, The Netherlands, 2006.

[17] Q. Yan, B. Huang, J. Yu, et al., "Intrinsic current-voltage characteristics of graphene nanoribbon transistors and effect of edge doping," Nano Letters, vol. 7, no. 6, pp. 1469-1473, 2007.

[18] J. W. G. Wildöer, L. C. Venema, A. G. Rinzler, R. E. Smalley, and C. Dekker, "Electronic structure of atomically resolved carbon nanotubes," Nature, vol. 391, no. 6662, pp. 59-62, 1998.

[19] R. A. Jishi, D. Inomata, K. Nakao, M. S. Dresselhaus, and G. Dresselhaus, "Electronic and lattice properties of carbon nanotubes," Journal of the Physical Society of Japan, vol. 63, no. 6, pp. 2252-2290, 1994. 
[20] R. F. Pierret, "Advanced semiconductor fundamentals," in The Modular Series on Solid State Devices, vol. 6, Prentice-Hall, Upper Saddle River, NJ, USA, 2nd edition, 2003.

[21] S. Datta, Quantum Transport Atom to Transistor, Cambridge University Press, Cambridge, UK, 2005.

[22] H. Zheng, Z. F. Wang, T. Luo, Q. W. Shi, and J. Chen, "Analytical study of electronic structure in armchair graphene nanoribbons," Physical Review B, vol. 75, no. 16, Article ID 165414, 6 pages, 2007.

[23] H. M. Tien, N. H. Chau, and P. T. K. Loan, "Tight-binding calculations of band structure and conductance in graphene nano-ribbons," Communications in Physics, vol. 19, no. 1, pp. $1-8,2009$.

[24] V. K. Arora, "Quantum engineering of nanoelectronic devices: the role of quantum emission in limiting drift velocity and diffusion coefficient," Microelectronics Journal, vol. 31, no. 1112, pp. 853-859, 2000.

[25] H. L. Hui, H. H. Ing, A. E. L. Chii, M. T. A. R. Ismail, and V. K. Arora, "The high-field drift velocity in degenerately-doped silicon nanowires," in Proceedings of the 2nd IEEE International Nanoelectronics Conference (INEC '08), Shanghai, China, March 2008.

[26] Q. Wan, J. Huang, A. Lu, and T. Wang, "Degenerate doping induced metallic behaviors in $\mathrm{ZnO}$ nanobelts," Applied Physics Letters, vol. 93, no. 10, Article ID 103109, 3 pages, 2008.

[27] M. T. Ahmadi, R. Ismail, M. L.P. Tan, and V. K. Arora, "The ultimate ballistic drift velocity in carbon nanotubes," Journal of Nanomaterials, vol. 2008, Article ID 769250, 8 pages, 2008. 

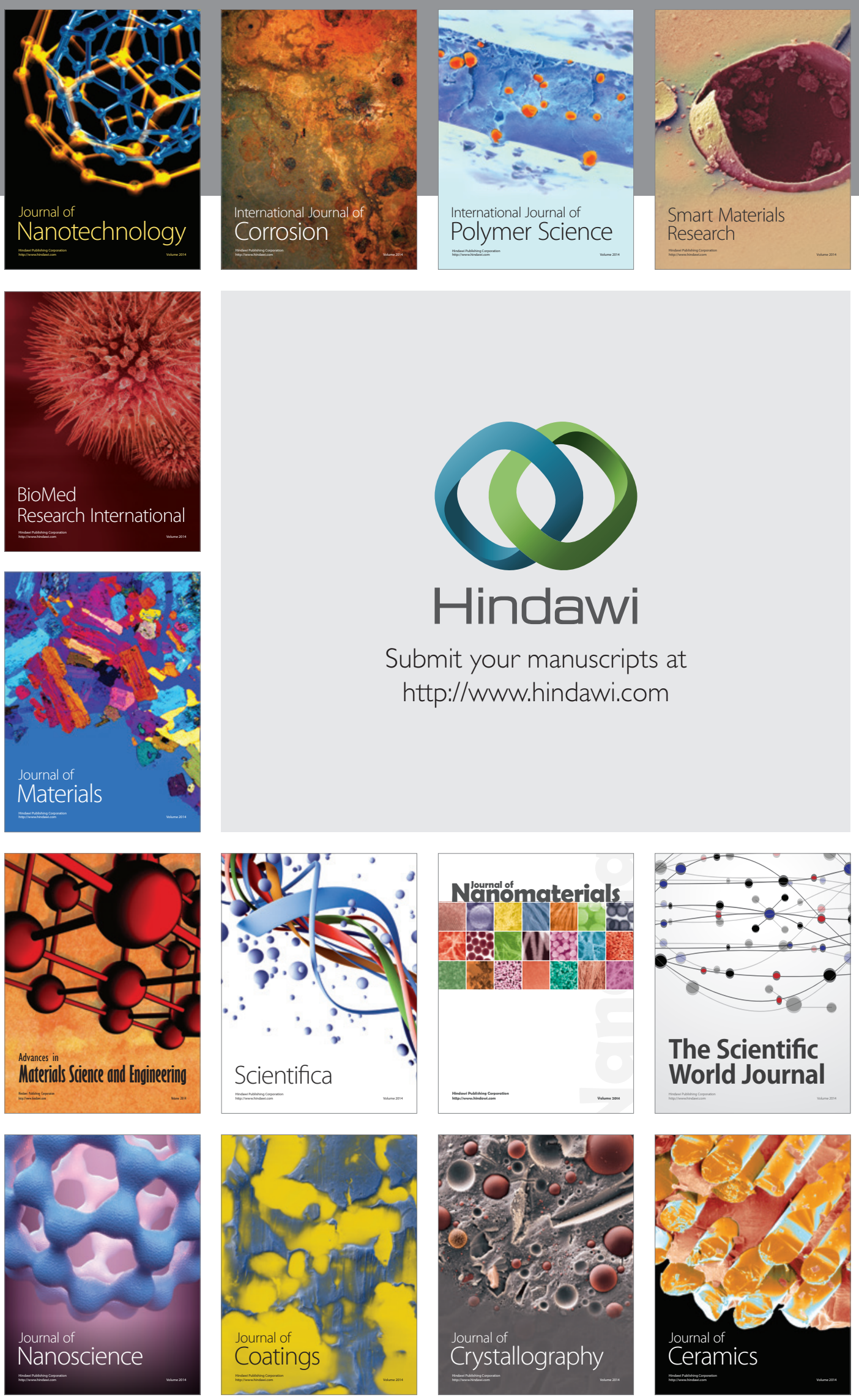

The Scientific World Journal

Submit your manuscripts at

http://www.hindawi.com

\section{World Journal}

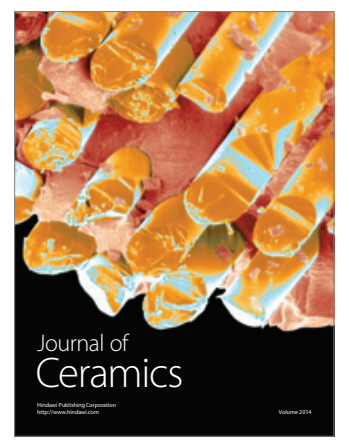

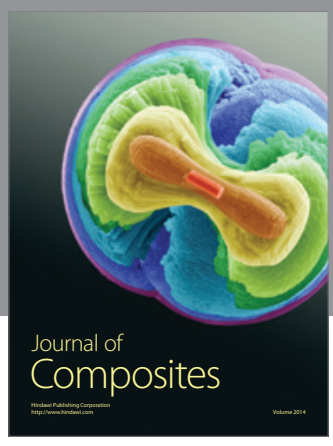
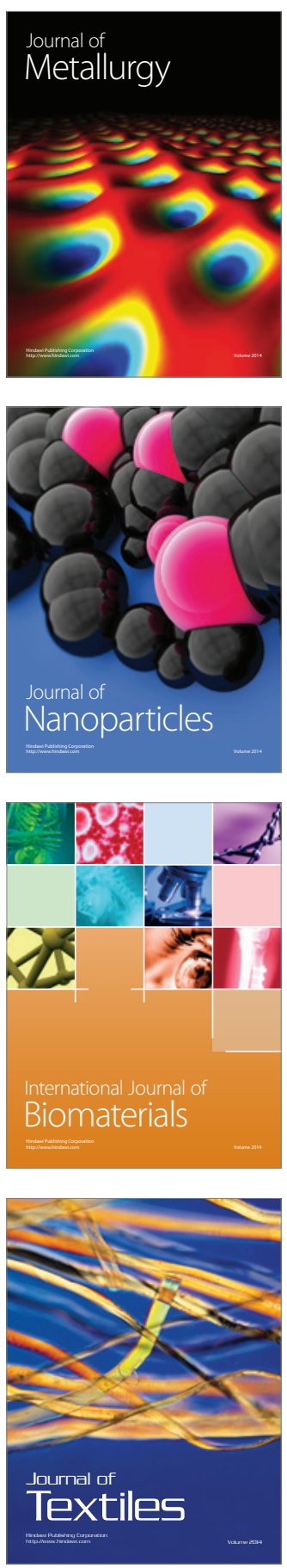